\title{
Off-label use of a medium-high molecular weight, isotonic and sterile hyaluronic acid in aesthetic medicine: Cases
} report

\author{
Svolacchia $\mathbf{F}^{*}$ and Svolacchia $\mathbf{L}^{2}$ \\ ${ }^{1}$ Department of Anatomical, Histological, Medical and Legal Sciences and the Locomotive Equipment, Section of Human Anatomy, Laboratory of Experimental \\ Morphology at the University of Rome "La Sapienza", Italy \\ ${ }^{2}$ Student, Medicine and Surgery, University of Rome "La Sapienza", Italy
}

\begin{abstract}
Improved lifestyle and increased interpersonal relationships have led older people to look for ways to improve their appearance and the aging signs of aging.

Objective: I studied a sterile and isotonic medical device based on hyaluronic acid in a variable solution between $1500 \mathrm{Kdalton}$ and $1800 \mathrm{Kdalton}$ in off label because it is dedicated to the use on mucous membranes at $0.12 \%$ concentration on sale since 2015 .

Methods: Subjective and objective evaluations were performed in vivo on selected voluntary patients, after having obtained regular informed consent.

Results: In agreement with previous studies on formulations containing hyaluronic acid with medium and high molecular weight specifically studied for aesthetic medicine, the results obtained in vivo demonstrate the effectiveness of this medium-high molecular weight formulated product injected into the dermis in reducing skin wrinkles and in improving the signs of aging.
\end{abstract}

\section{Introduction}

The improvement of interpersonal relationships combined with the extension of life expectancy have led to a significant increase in the overall appearance of the face and body [1]. The increasing proportion of women and men interested in skin rejuvenation has created a huge demand for so-called anti-aging remedies to rejuvenate the photoaging skin or naturally aging in the shortest possible time. The signs of the relative skin damage associated with age, such as wrinkles, relaxation and irregular pigmentation, are in fact strongly influenced by environmental factors, in particular sun exposure during life [2]. The young fibroblasts first produce elastin then glycosaminoglycans and eventually undergo involution through the normal aging process for the induction of reactive oxygen species [2,3]. Furthermore, the induction of the common deletion in human skin fibroblasts is accompanied by a measurable decrease in oxygen consumption and an increase in metalloprotein-1. Many studies have shown, in fact, how the induction of matrix metalloproteinases plays a major role in the pathogenesis of photoaging [4,5]. As a consequence aged skin displays an array of fine, superficial facial lines that leads to the formation of deep creases over the forehead and between the eyebrows, periorbitally, and in the nasolabial folds (Photo1). During the past few years, many different techniques for rejuvenation have been proposed. The simplest procedures involve the use of hyaluronic acid in variable concentrations in order to have a rapid remodeling of wrinkles and improve the survival of fibroblasts through a suitable cross-talking with growth factors [6], the most complex ones involve the association of several active ingredients in order to activate also the cellular metabolism and the protection of the membranes $[7,8]$.

\section{Project}

Hyaluronic acid is an important component of the extracellular matrix and helps to give elasticity to the skin. It has a key position in wound healing and tissue repair processes due to its ability to maintain a moist environment conducive to healing and stimulation of growth factors, cellular constituents and migration of various cells essential for healing [9]. New production of collagen and elastin appear to be induced by degradation of injected hyaluronic acid as a response of fibroblasts on the matrix [10] and its use is safe [11]. Its depletion contributes to the appearance of wrinkles and blemishes (Figure 1). Hyaluronic acid (HA) is a natural linear polysaccharide that has been widely used in the biomedical field as it is a biocompatible, biodegradable, non-toxic and non-immunogenic polymer with high water affinity [12].

The product used for this study is a medium-high molecular weight hyaluronic acid of 1500 Kdalton and 1800 Kdalton at $0.12 \%$ concentration. It has been specifically formulated and marketed by a pharmaceutical company for use on mucous membranes. The concentration is usually expressed in weight / volume and in this case means that by weight its concentration is $1.2 \mathrm{mg} / \mathrm{ml}$. Before using

${ }^{*}$ Correspondence to: Fabiano Svolacchia, Department of Anatomical, Histological, Medical and Legal Sciences and the Locomotive Equipment, Section of Human Anatomy, Laboratory of Experimental Morphology at the University of Rome "La Sapienza", Italy, E-mail: fabiano.svolacchia@uniroma1.it

Key words: antiaging, signaling molecules, photo-aging, biostimulation, chronoaging

Received: March 28, 2018; Accepted: April 17, 2018; Published: April 21, 2018 
the product, I compared it with others specifically formulated by a well-known cosmetic medicine company to evaluate the percentage of hyaluronic acid in them. I observed that in the formulations commercialized the percentage ranged from $0.01 \%$ to $1.5 \%$ and this has reassured me about the possibility of its use in mesotherapeutic antiaging treatments, albeit in off-label. Naturally, the product had to be safe, biocompatible and stable at the implant site, with possible minimal complications. The sterility and the isotonicity of the formulation pushed me to an off-label use as an adjuvant in anti-aging treatments in aesthetic medicine.

High molecular weight HA (HMWHA) is deposited in normal tissues during homeostasis and promotes their stability whereas low molecular weight HA fragments (LMWHA) may arise from enzymatic activities. The degradation of HMWHA to LMWHA fragments, often leads to the generation of biologically active oligosaccharides with different properties [13]. Native high molecular weight HA has structural properties whereas HA degradation products (oligomers) stimulate endothelial cell proliferation and migration. HA oligomers modulate inflammatory processes and promote neo-angiogenesis during the different steps of wound healing. HA mediates its biological effects through binding interactions with specific cell-associated receptors (CD44 and RHAMM) [14]. MW HA may promote the biosynthesis of growth factors such as FGF-2 and KGF [15].

\section{Aim}

The aim of the study was to evaluate the rejuvenation effects of a medium-high molecular weight sterile and apyrogenic hyaluronic acid used in off-label and injected into the dermis on subjects affected by chrono and photo-aging in order to allow the doctor to reevaluate his working methods.

\section{Material and methods}

Each $5 \mathrm{ml}$ sterile ampoule contained $0.12 \%$ hyaluronic acid in physiological solution and water for injections. The study took place in vivo and the safety and efficacy of the product was established to reduce the overall appearance of thin and deep lines.

\section{Patient enrollment}

Before the study, each subject was informed about the purpose of the study, and their written informed consent was obtained according to the ethics of medical-device experimentation. No drugs or cosmetic procedures affecting the course of the antiaging treatment were allowed 2 weeks prior to the study period.

All the 28 volunteer women (age range 40-55 years) between October 2017 and January 2018 attended the medical office. The only criterion for entry in the study was the presence of one or more signs of chrono or photoaging affecting the face, such as fine wrinkling around the eyes, crease lines around the mouth and cheeks, wrinkling and spots on the back of the hands, etc, corresponding to degrees 3-5 of the photodigital scale described by Larnier et al. [16]

\section{In vivo studies}

Patient enrollment: The possibility of having a single product composed of medium-high molecular weight hyaluronic acid can improve patient-medical compliance and for the personalization of the active ingredients to be associated. If the doctor then wanted to combine this composition with other active ingredients could exploit the pharmacological activity of hyaluronic acid with the metabolic activity on fibroblast through the different pharmacological activities at different levels, both cellular and matrix and would help the operator to obtain the best rejuvenation results from skin biostimulation. This hypothesis will be part of a further study

Injection technique: The contents of the ampules were injected directly into different skin areas of the 28 submissive women, mean age 46.7 years. The puncture technique used was based on a single injection every 7 day for 4 weeks, followed by other injections for a further 2 weeks for a 60-day personalized global treatment with a final control at day 90 (regression period).

The treatment used was based on the technique of mesotherapy, using $2.5 \mathrm{ml}$ of solution and a $30 \mathrm{~g}$ needle positioned at $45^{\circ}$ to the surface of the skin. The needle was inserted up to the medium subcutis, suction was performed to ensure that the tip was not inside a blood vessel and the injection was then started slowly when the needle was withdrawn. The injection speed was always lower than $0.3 \mathrm{ml} / \mathrm{min}$. The firm massage, with the index finger inside the mouth and the thumb outside, was then used to remove any irregularities. The amount of 2.5 $\mathrm{ml}$ is sufficient to treat the entire face. With each vial it was possible to treat two patients.

\section{Control assessment and dermatological evaluation}

Control visits and evaluations were undertaken on the first day (D1, baseline) and after 60 (D60) days of treatment, with a followup visit at D90 (regression period). The individual signs of chrono or photo-aging symptoms of skin irritation and the degree of the obtained correction for each treatment and each area were evaluated objectively using a $0-10$ visual analog scale with separate scores for each site of the face $(0=$ no correction; $5=$ satisfactory correction; 10 $=$ total correction). The degree of satisfaction with the efficacy of the product was also obtained subjectively by asking the patients if there was any itching, stinging, or burning sensation. The different mean evaluations are reported in (Figure 1).

\section{Subjects evaluation}

After the first and second month of treatment, with a follow-up at the third month (regression period), the subjects evaluated their satisfaction or dissatisfaction by giving scores on firmness, softness, hydration, and wrinkle appearance, using a scale of 0-4 for each criterion ( 0 = unsatisfactory; 4 = satisfactory), as per Berardesca et al. [17] The obtained results are reported in (Figure 2).

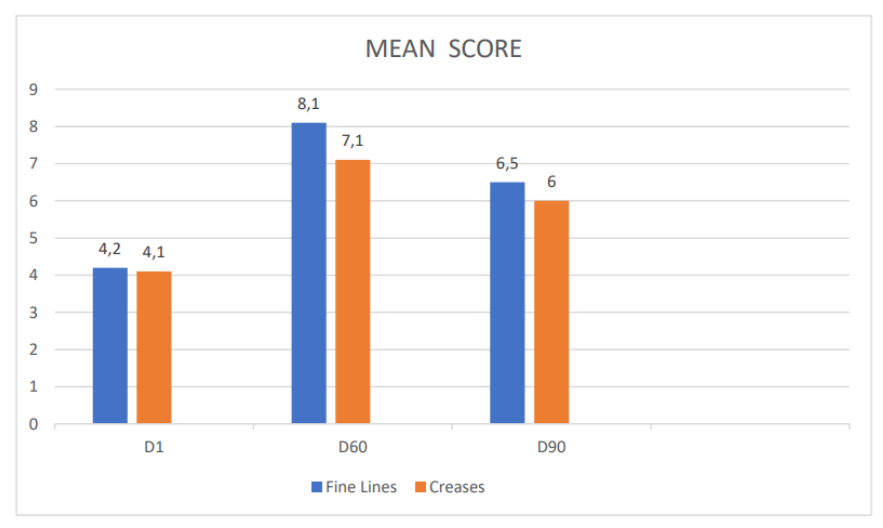

Figure 1. Dermatological mean evaluation on signs of chrono or photo-ageing after injective treatment 


\section{Results and discussion}

The obtained results seem to be in line with our expectations in vivo evaluations on the efficacy of the treatment. Practically all the subjects treated during the 60-day period reported that they were satisfied with the general aspect of their skin, which appeared softer and more hydrated since the first month of treatment, as shown in (Figures 3 and 4). In line with their self-evaluation, the appearance of the fine wrinkling was notably reduced and the consequent skin softness and firmness enhanced during the entire treatment period. This general amelioration remained during the regression period also, 30 days after the interruption of the treatment. As shown in (Figure 2), both fine wrinkling and crease lines were reduced soon after the first 15 days of treatment, so that the general appearance of the face was notably ameliorated during the regression period also.

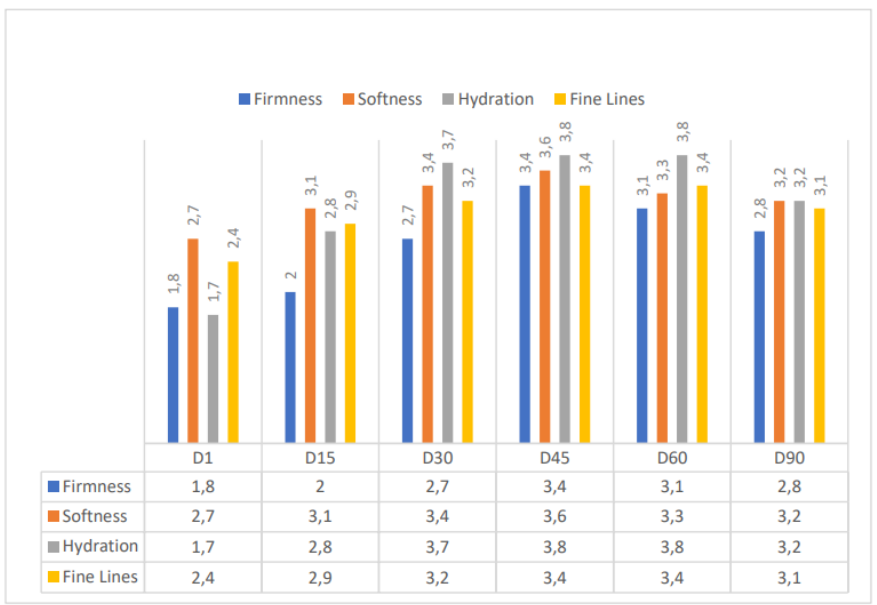

Figure 2. Self-evaluation and satisfaction of the subjects treated (general degree of satisfaction).

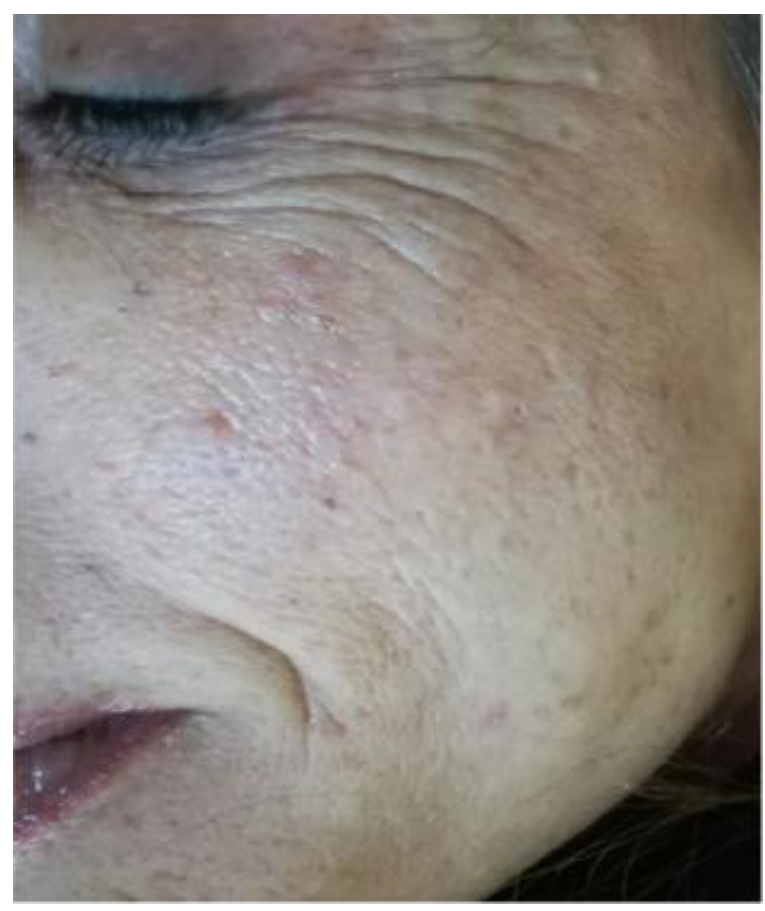

Figure 3. Signs of chrono or photo-ageing after injective treatment

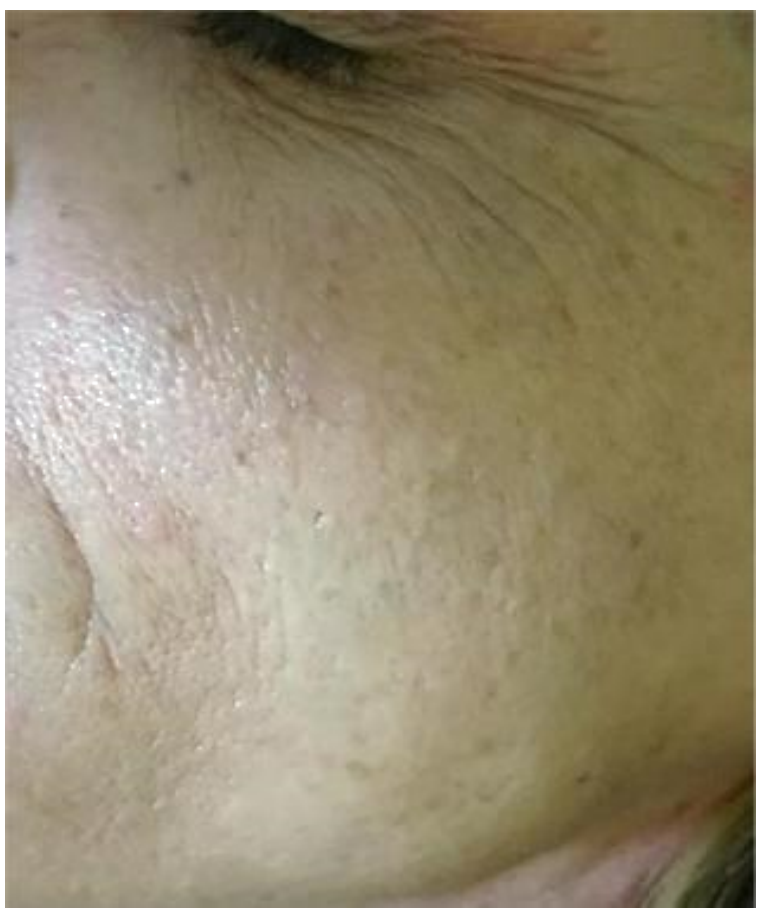

Figure 4. Self-evaluation and satisfaction of the subjects treated

\section{Conclusion}

This interesting general amelioration is shown in (Figure 2), where a reduction of fine lines is evident. The in vivo results are confirmed based on our findings, the jaluronic acid active ingredient used, seems to be useful in improving the activity of fillers, rendering it useful as an antiaging remedy for the aestethic medicine armamentarium. In conclusion, this simple and low-cost biostimulating medical device, can be used off-label, for wrinkle treatment and rejuvenating looks. I am continuing the research using this hyaluronic acid as a base for other active ingredients such as amino acids, vitamin $\mathrm{C}$ and vitamin $\mathrm{D}$ and antioxidant.

\section{Reference}

1. Kramer U, Schikowski T (2006) Recent demographic changes and consequences for dermatology. In: Gilchrest BA, Krutman J, editors. Skin Aging. New York: SpringerVerlag USA: 1-8.

2. Krutmann J, Gilchrest BA (2006) Photoaging of skin. In: Gilchrest BA, Krutman J, editors. Skin Aging. New York: Springer-Verlag, USA: 33-43.

3. Jacobs HT (2003) The mitochondrial theory of aging: dead or alive? Aging Cell 2 11-17. [Crossref]

4. Fisher GJ, Wang ZQ, Datta SC, Varani J, Kang S, et al. (1997) Pathophysiology of premature skin aging induced by ultraviolet light. N.Engl J Med 337: 1419-1428. [Crossref]

5. Schraffeter-Kochanek K, Brenneisen P, Wenk J (2000) Photoaging of the skin from phenotype to mechanisms. Exp Gerontol 35: 307-316. [Crossref]

6. Morganti P (2009) Beauty from the inside and the outside. Natural products work in multiple ways. In: Tabor A, Blair R, editors. Nutritional Cosmetics: Beauty from Within. New York: William Andrew, USA: 95-111.

7. Pierfrancesco Morganti, Paolo Palombo, Marco Palombo, Giuseppe Fabrizi, Antonio Cardillo (2012) "A phosphatidilcoline hyaluronyc acid chitin-nanofibrils complex for a fast skyn remodelling and rejuvenating look ". Clinical, Cosmetic and Investigational Dermatology 5: 213-20. [Crossref]

8. Palmieri A, Avantaggiato A, Cura F, Casale M, Lopez MA, et al. (2017) Biorevitalization: an in vitro study on gingival fibroblast. $J$ Biol Regul Homeost Agents 3: 147-153. [Crossref] 
9. Keen MA (2017) Hyaluronic Acid in Dermatology. Skinmed 1: 441-448. [Crossref]

10. Kavasi RM, Berdiaki A, Spyridaki I, Corsini E, Tsatsakis A, et al. (2017) HA metabolism in skin homeostasis and inflammatory disease. Food Chem Toxicol 101: 128-138.

11. Neuman MG, Nanau RM, Oruña-Sanchez L, Coto GJ (2015) Hyaluronic acid and wound healing. Pharm Pharm Sci 18: 53-60.

12. Larraneta E, Henry M, Irwin NJ, Trotter J, Perminova AA, et al. (2018) Synthesis and characterization of hyaluronic acid hydrogels crosslinked using a solvent-free process for potential biomedical applications. Carbohydr Polym 181: 1194-1205. [Crossref]

13. Landau M, Fagien S (2015) Science of Hyaluronic Acid Beyond Filling: Fibroblasts and Their Response to the Extracellular Matrix. Plast Reconstr Surg. 136: 188S-195S. [Crossref]

14. Gall Y (2010) [Hyaluronic acid: structure, metabolism and implication in cicatrisation]. Ann Dermatol Venereol 137: S30-S39.

15. Park JU, Tsuchiya T (2002) Increase in gap junctional intercellular communication by high molecular weight hyaluronic acid associated with fibroblast growth factor 2 and keratinocyte growth factor production in normal human dermal fibroblasts. Tissue Eng 8: 419-27. [Crossref]

16. Larnier C, Ortonne JP, Venot A (1994) Evaluation of cutaneous photodamage using a photographic scale. Br J Dermatol 130: 167-173. [Crossref]

17. Berardesca E, Distante F, Anthoine P, Rabbiosi G, Aubert L(1997) Clinical and instrumental evaluation of the activity of an anti-wrinkle product on cutaneous relief and photoaged skin. J Appl Cosmetol 15: 69-75.

Copyright: (C2018 Svolacchia F. This is an open-access article distributed under the terms of the Creative Commons Attribution License, which permits unrestricted use, distribution, and reproduction in any medium, provided the original author and source are credited. 